\title{
Manipulation of laser-generated energetic proton spectra in near critical density plasma
}

\author{
Charlotte A. J. Palmer ${ }^{1,2} \dagger$, Nicholas P. Dover ${ }^{2}$, Igor Pogorelsky ${ }^{3}$, Matthew J. \\ V. Streeter ${ }^{1}$ and Zulfikar Najmudin ${ }^{2}$ \\ ${ }^{1}$ Deutsches Elektronen-Synchrotron (DESY), D-22607 Hamburg, Germany \\ ${ }^{2}$ The John Adams Institute for Accelerator Science, The Blackett Laboratory, \\ Imperial College London, London, SW7 2AZ, UK \\ ${ }^{3}$ Accelerator Test Facility, Brookhaven National Laboratory, P.O. Box 5000, Upton NY 11973, USA
}

(Received 2 May 2014; revised 13 August 2014; accepted 18 August 2014; first published online 10 October 2014)

We present simulations that demonstrate the production of quasi-monoenergetic proton bunches from the interaction of a $\mathrm{CO}_{2}$ laser pulse train with a near-critical density hydrogen plasma. The multi-pulse structure of the laser leads to a steepening of the plasma density gradient, which the simulations show is necessary for the formation of narrow-energy spread proton bunches. Laser interactions with a long, front surface, scale-length $\left(\gg c / \omega_{p}\right)$ plasma, with linear density gradient, were observed to generate proton beams with a higher maximum energy, but a much broader spectrum compared to step-like density profiles. In the step-like cases, a peak in the proton energy spectra was formed and seen to scale linearly with the ratio of laser intensity to plasma density.

\section{Introduction}

The generation of energetic ion beams from the interaction of intense lasers with plasma targets is a highly active area of research due to promising experimental results that have great implications for potential applications (Hatchett et al. 2000; Key 2007; Ter-Avetisyan et al. 2008). Much of the work to-date has been clearly summarised by Daido et al. (2012) and Macchi et al. (2013). Sheath acceleration (Wilks et al. 2001; Mora 2003; Schreiber et al. 2006), also termed target normal sheath acceleration, and radiation pressure acceleration (Esirkepov et al. 2004; Robinson et al. 2008) have provided key areas of exploration. The majority of experimental work has resulted in the production of ion beams with maximum energies of $10 \mathrm{~s}$ of $\mathrm{MeV}$ and approximately thermal energy distributions, a feature of sheath acceleration (Hatchett et al. 2000; Snavely et al. 2000). In this mechanism, the laser field heats electrons which then stream through the target and establish an electrostatic sheath field at the rear surface. This field is sufficient to ionise the target and accelerate the resulting ions away from the surface. The broad energy spectra of the resultant beams is undesirable for a number of potential applications which require narrow, or controlled energy spread, e.g. hadron therapy (Bulanov and Esirkepov 2002). Specialised targets (Hegelich et al. 2006; Schwoerer et al. 2006) and energy filtering (Toncian et al. 2006) could be used as a means to reduce the energy spread, although this also diminishes the charge available for the application. 
Alternately, radiation pressure acceleration has been proposed as a means of achieving narrow energy spread ion beams without filtering. Here, the acceleration is driven by the radiation pressure of the incident laser pulse which sets up an accelerating electrostatic field at the laser-plasma boundary. For thick targets ( $>$ $c / \omega_{p}$ ), hole-boring (Wilks et al. 1992; Robinson et al. 2009) occurs, during which ions within the bulk plasma are reflected from the inwardly propagating density front moving with velocity, $v_{h b}$. Macchi et al. (2005) proposed an alternative model for hole-boring acceleration, in which ions are dragged from behind the hole-boring front by the electrostatic field which accelerates them in the forwards direction. For thin targets, the influence of the incident laser field penetrates throughout the target, which can then be accelerated as a whole (Esirkepov et al. 2004) and has been termed light-sail acceleration (Robinson et al. 2008). The resultant energy spread is peaked at a value $\left(E_{i}\right)$ which scales with laser intensity $\left(I_{L}\right), E_{i} \propto I_{L}$ for hole-boring, and $E_{i} \propto I_{L}^{2}$ for light-sail as compared with the sheath acceleration mechanism for which $E_{i} \propto \sqrt{I_{L}}$ (Mora 2003).

Denavit (1992) and Silva et al. (2004) describe the generation of proton beams through acceleration by a collisionless electrostatic shock. The shock is launched by an 'ion-piston' pushing against an overdense plasma target. Ions within the bulk plasma can be reflected from this shock up to twice the shock velocity, dissipating the energy of the shock. Fiuza et al. (2012) demonstrates the possibility of achieving narrowenergy spreads through this mechanism. Both hole-boring and shock acceleration are the reflection of ions from a moving potential generated either directly via radiation pressure, or indirectly driven by the laser via electron heating. The velocity of this potential is strongly dependent on the plasma temperature and density, as well as the laser intensity (Denavit 1992; Zhang et al. 2007).

Theoretical work into light-sail ion acceleration has demonstrated the potential to deliver high-quality, high-energy beams in ideal conditions. However, it has proven very difficult to realise these results due to practical challenges such as targetry and control of instabilities (Palmer et al. 2012). More positive results have been obtained within the parameter space of hole-boring and shock acceleration (Palmer et al. 2011; Haberberger et al. 2012). For these experiments long wavelength lasers $\left(\lambda_{L} \approx 10 \mu \mathrm{m}\right)$ have been used with near-critical density plasmas, where critical density $\left(n_{c r}\right)$ is defined as the density at which the laser and plasma frequencies are equal, to obtain $\mathrm{MeV}$ beams with small ( $<10 \% \mathrm{rms}$ ) energy spread. A linear scaling of $E_{i} \propto I_{L} / n_{e}$ has also been observed.

Here, we present results from simulations performed to model the experimental conditions present during the investigations conducted by Palmer et al. and to provide illumination as to the formation of the narrow energy spread beams. The experiment used a $\mathrm{CO}_{2}$ laser to produce a pulse train with total energy $\approx 3 \mathrm{~J}$, split into multiple 6 ps pulses, achieving a peak value of $a_{0} \approx 0.5$. Auxiliary details of the experimental arrangement and results are available in Najmudin et al. (2011); Palmer et al. (2011) with further details of transverse probing through the overdense target in Pogorelsky et al. (2012). Subsequently to this experiment, further experiments utilising a $\mathrm{CO}_{2}$ pulsed laser, together with a near-critical density hydrogen gas jet, also reported the measurement of narrow energy spread peaks within the proton spectra (Haberberger et al. 2012). This experiment utilised a linearly polarised laser with a higher total energy $(\approx 20 \mathrm{~J})$ spread over a duration of $100 \mathrm{ps}$ in multiple 3 ps pulses. A peak value of $a_{0}$ between 1.5 and 2.5 was reported. Despite the common observation of narrow energy spread features the two experiments exhibit some key differences. In the work of Haberberger et al. (2012), the acceleration was attributed to reflection from a 
collisionless shock formed by the electrons within the low density plasma in the ramp that are efficiently heated by one of the linearly polarised, incident laser pulses. In contrast Palmer et al. (2011) presents an interaction with a circularly polarised pulse. Circular polarisation leads to reduced heating of the plasma electrons and, in this instance, the radiation pressure of the laser plays an important role in the interaction. Radiation pressure results in compression of the front surface of the plasma forming a density front which propagates into the target. The density front has an associated electric field due to the large density and pressure discontinuity with the background plasma, which is much cooler than that of Haberberger et al. (2012), resulting in a shock which reflects ions to twice the front velocity with higher reflectivity (Dover et al. 2012). The density front continues to propagate into the target after the laser loses influence due to the momentum associated with the front. It continues to accelerate particles from the bulk of the plasma by electrostatic reflection until it eventually dissipates or it reaches the rear boundary of the plasma. As shown in the simulations presented here, these two different regimes can be assessed by variations in the scale-length of plasma with which the laser interacts.

\section{Simulation set-up}

The PIC code OSIRIS (Fonseca et al. 2002) was used to perform 2D3V simulations with a spatial resolution of $33 \mathrm{~nm} /$ cell in the laser propagation direction $(x)$, and $176 \mathrm{~nm} /$ cell in the transverse direction $(y)$, with a window size of $468 \times 500 \mu \mathrm{m}$.

The target was modelled as a cold $\left(T_{e}=0\right)$, fully-ionised, hydrogen plasma $\left(\mathrm{H}^{+}\right)$ with one particle per cell for the ion species and four particles per cell to represent the electrons. This number was limited due to computational constraints and results in an increase in particle noise. However, as the grid resolution was sufficient to accurately resolve the skin depth of the plasma, this does not have significant effect on the results. Characterisation of the gas jet target used in the experiments revealed a triangular density profile (Najmudin et al. 2011). Therefore, a set of simulations were performed with a triangular profile rising from zero to peak density over $60 \mu \mathrm{m}$.

The laser pulse was circularly polarised with wavelength of $10 \mu \mathrm{m}$. The gaussian temporal profile was approximated using a 5th order polynomial with FWHM of 3 ps. The pulse was focussed to a gaussian spot of $74 \mu \mathrm{m}$ (FWHM) corresponding to the experimentally determined spot size. The value of $a_{0}$, the peak normalised vector potential of the laser, was set to 1.8 with the peak intensity arriving at the target surface at $t \approx 5 \mathrm{ps}$. This is higher than the experimental value of $a_{0}$ as preliminary simulations indicated that the level of self-focusing in 2D simulations would not accurately represent the increase of intensity occurring in three dimensions.

In the work of Palmer et al. (2011), the pulse train of the $\mathrm{CO}_{2}$ laser was found to be often dominated by two major pulses, though the exact pulse structure was found to vary from shot to shot. A simulation was performed in which two identical, temporally-separated, laser pulses were incident upon a plasma with the previously described triangular density profile. The peak-to-peak separation of the pulses was 8 ps. This enabled the interaction of both pulses to be observed within the computational limits and revealed a steepening of the surface density gradient during the interaction with the first pulse. The second pulse was then incident upon an approximately step-like density profile rather than a triangular one. Sharp plasma density fronts were also observed experimentally by simultaneous transverse shadowgraphy and interferometry (Palmer et al. 2011; Dover et al. 2012; Pogorelsky et al. 2012). 

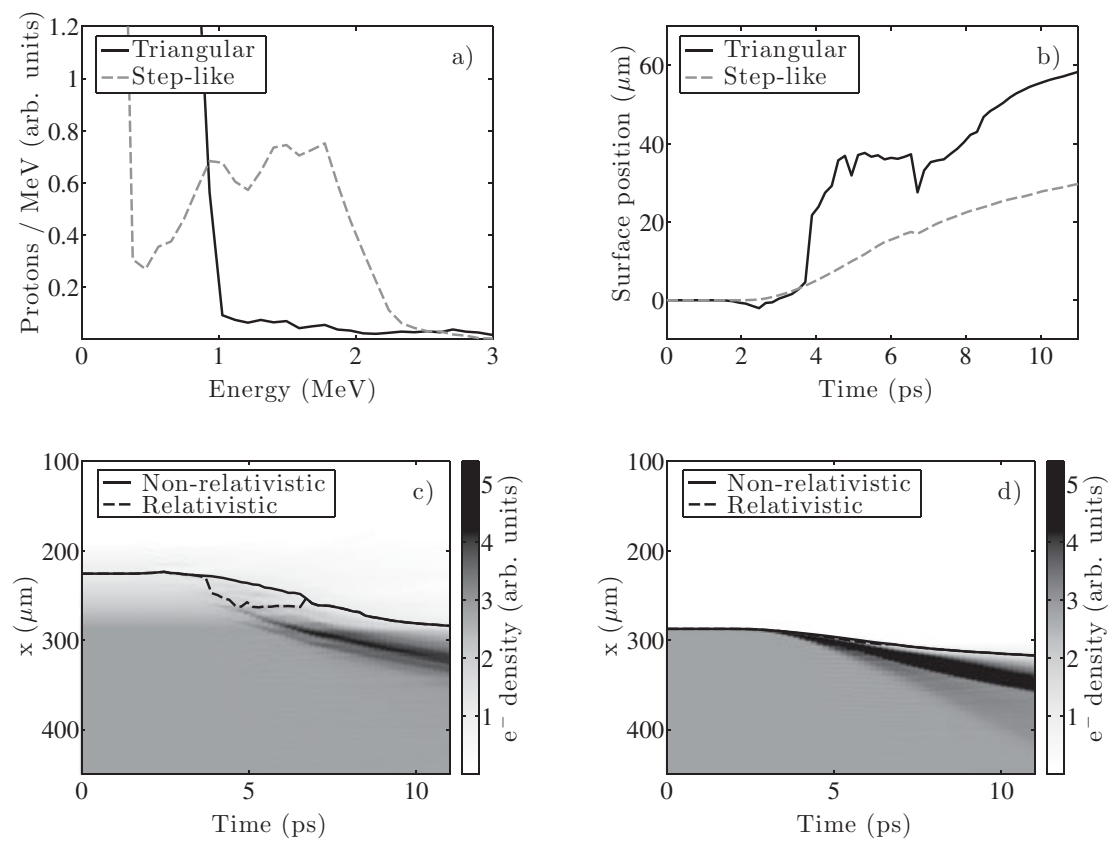

FIGURE 1. For targets with either triangular or step-like density profile and peak density of $2.8 n_{c r}$, irradiated with a peak intensity corresponding to $a_{0}=1.8$ : a) Proton spectra $(t=10 \mathrm{ps})$ including protons within a $10 \mu \mathrm{sr}$ solid angle around the laser axis. b) Axial position of the relativistic critical surface with time. c) Axial density map with time for the triangular density profile target. d) Axial density map with time for the step-like density profile target. Both c) and d) also show the non-relativistic and relativistic critical surface positions.

As a consequence of the simulated density profile steepening, a further set of simulations were performed to compare the effect of triangular and step-like density profiles on the acceleration of ions. For the step-like density profile, the density rose from vacuum to maximum over a distance of $0.2 \mu \mathrm{m}$. The maximum density was varied in the range $n_{e}=1-7.5 n_{c r}$.

\section{Effect of plasma density profile at the interaction surface}

The formation of a density front and the recession of the surface were observed in both simulations with a step-like and a triangular density profile. However, peaked spectra, comparable with those measured during the experiment, were only obtained in the step-like case (Fig. 1(a)).

Figure 1(c) presents the evolution of the axial electron density profile for a target with an initially triangular profile with peak density of $2.8 n_{c r}$. The position of both the non-relativistic and relativistic critical density surfaces as a function of time, are indicated by the overlaid lines. When the laser enters the underdense plasma, it starts to perturb the plasma and create density ripples. The majority of the laser pulse energy reaches the critical density surface and the radiation pressure causes the laserplasma boundary to recede. As the laser intensity increases and becomes relativistic, the laser propagates up the shallow density gradient to beyond the non-relativistic critical surface. At $t=5 \mathrm{ps}$, corresponding to the arrival of the peak of the laser intensity, a density spike is formed at the surface for which $n_{e}=\sqrt{\left(1+a_{e n v}(t)^{2}\right)} n_{c r}$ 

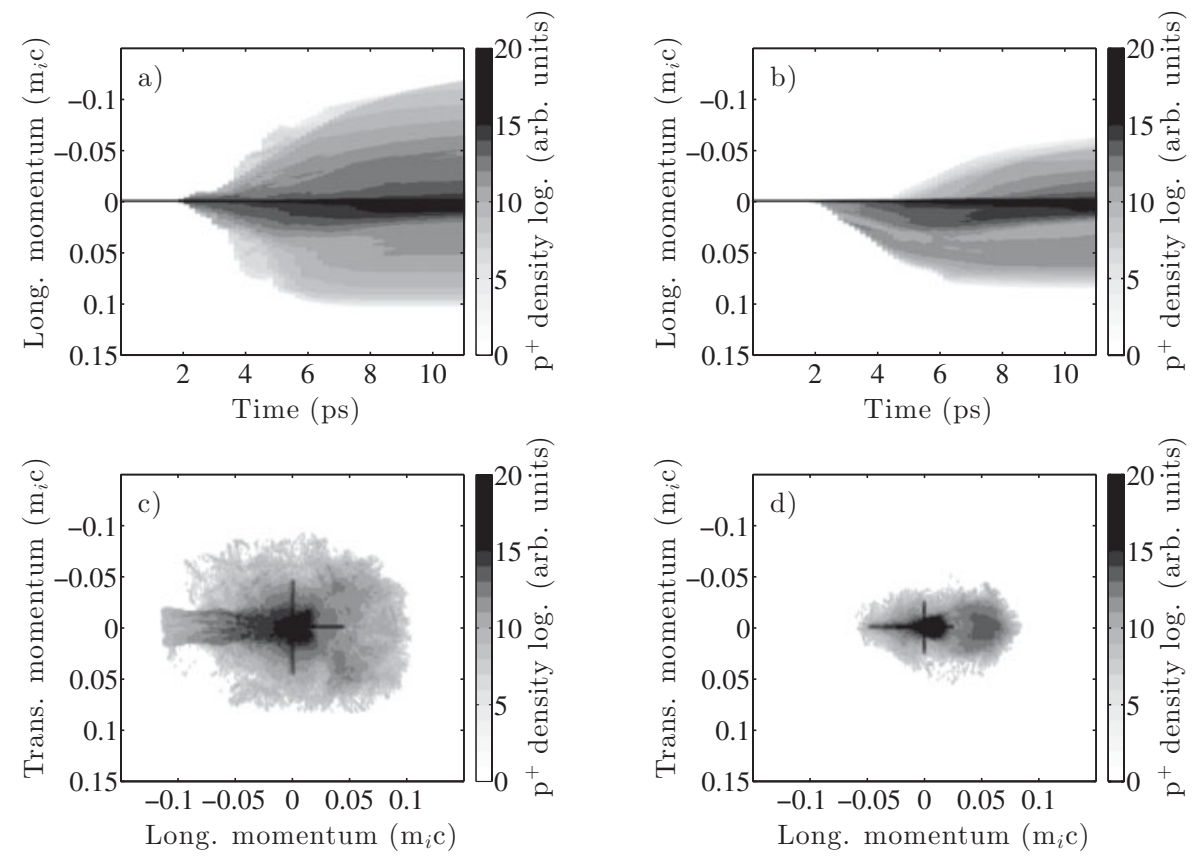

FIGURE 2. Evolution of longitudinal proton momentum for the interaction of an $a_{0}=1.8$ laser pulse and (a) a target with a triangular density profile, (b) a target with a step-like density profile, both with a peak density of $2.8 n_{c r}$. Positive momentum is along the direction of laser propagation. $p_{x} p_{y}$ phase-space of the protons at $t=10 \mathrm{ps}$ for $(\mathrm{c})$ the target with a triangular density profile, and (d) the target with a step-like density profile.

i.e. at the relativistically correct critical surface. Here, $a_{e n v}(t)$ is the amplitude of the normalised vector potential at time $t$. This front then propagates inwards, away from the influence of the laser field.

Figure 1(d) presents the evolution of the axial electron density profile for the steplike target, with peak density of $2.8 n_{c r}$. Here it can be seen that there is minimal divergence of the non-relativistic and relativistic critical surface and that a steepgradient, high-density front is formed which, as in the triangular case, propagates into the target beyond the critical surface.

In both cases, the density front is formed during the interaction with the laser, and the velocity of the front is linked to the velocity of the critical surface at this time. Figure 1(b) provides a comparison of the surface motion for the two targets showing that the velocity of the front is more variable in the triangular case than the step-like case. Although the hole-boring front is generated during the interaction with the laser, as the radiation pressure of the laser decreases and the critical surface begins to expand the density front continues to propagate into the target as a shock. This can also be seen by studying the evolution of the ion momentum.

Figures 2(a) and (b) present the longitudinal ion momentum against time for the triangular and step-like targets respectively. Here, positive momentum is in the laser propagation direction. In the triangular case, low density plasma in the density ramp is rapidly heated and expands towards the incoming laser. As the laser intensity increases towards its maximum, there is a rapid increase in forward momentum. This occurs when relativistic transparency is observed and the laser rapidly penetrates into the 
up-slope of the plasma profile (Fig. 1(c)). The protons accelerated during this period exhibit a broad energy spread. The laser pulse ends at $t=8 \mathrm{ps}$, after which there is no further acceleration of protons. At late times, it can be seen that the forward momentum of the bulk of the ions reduces and that the maximum momentum of the ion population no longer increases. However, the underdense plasma continues to expand driven by thermal pressure of the hot plasma.

For the step-like target, it can be seen that the radiation pressure of the laser inhibits expansion of the front surface of the target. Without the density ramp, the laser energy is not coupled as efficiently to the plasma as in the triangular case, and therefore the laser radiation pressure overcomes the thermal pressure of the plasma. There is relatively constant velocity in the forward direction, although the protons are quickly seen to separate into two populations. The higher energy population maintains its maximum energy after the laser pulse. Although the energy of the spectral peak is seen to broaden it remains distinct from the lower energy population. The central momentum of this high energy population, $p_{\text {peak }}$, taken at $t=10 \mathrm{ps}$ is $0.055 m_{i}$ c, corresponding to a velocity of approximately $16 \mu \mathrm{m} / \mathrm{ps}$ (and proton energy of $1.4 \mathrm{MeV}$ ). This is approximately 3 times higher than the maximum velocity that is reached by the critical surface at $t=6 \mathrm{ps}$.

In the triangular case the maximum momentum is $p_{\max }=0.087 m_{i} c$ corresponding to a velocity of $26.1 \mu \mathrm{m} / \mathrm{ps}$ (and proton energy of $3.5 \mathrm{MeV}$ ). Comparing this with the maximum velocity of the critical surface, it is also possible that these high energy ions could be accelerated by reflection from this front, indeed the acceleration of this high energy population appears closely related to the velocity of the critical surface and experiences the greatest acceleration at the point of the spike in velocity of the front.

Figures 2(c) and (d) present the longitudinal and transverse momenta at $t=10 \mathrm{ps}$. The high density lines visible along the horizontal and vertical axes are due to sheath acceleration caused by simulation-box boundary effects at the edges of the simulation box which are in contact with the plasma. From these plots, it can be seen that the momentum peak observed in the spectrum of the step-like target is directed along the laser propagation direction. In contrast the triangular target shows isotropic emission, with the exception of strong backward acceleration within the low density plasma in the density ramp, and two spectral enhancements at $\pm 45^{\circ}$ to the forward direction. However, the experimental proton spectrum measurements were taken through an onaxis pinhole covering a solid angle of $10 \mu \mathrm{sr}$, which would have prevented observation of the off-axis population and acted to accentuate the on-axis peak in the proton spectra. Also the on-axis flux in the triangular density profile case is much lower than in the step-like case, so the observed signal would be expected to be much lower over the entire observable energy range.

\section{Effect of variation of target density}

Generation of separate high-energy, ion populations was also observed in steplike targets with increasing maximum density up to $7.5 n_{c r}$. The acceleration of this population can be seen very clearly in Fig. 3(a). This presents the longitudinal momentum evolution for a target with a maximum density of $3.8 n_{c r}$. Here, a population of rapidly accelerating ions is generated shortly after the arrival of the laser and the recession of the critical surface. The acceleration of this high-energy population is approximately twice that of the bulk of the plasma. 

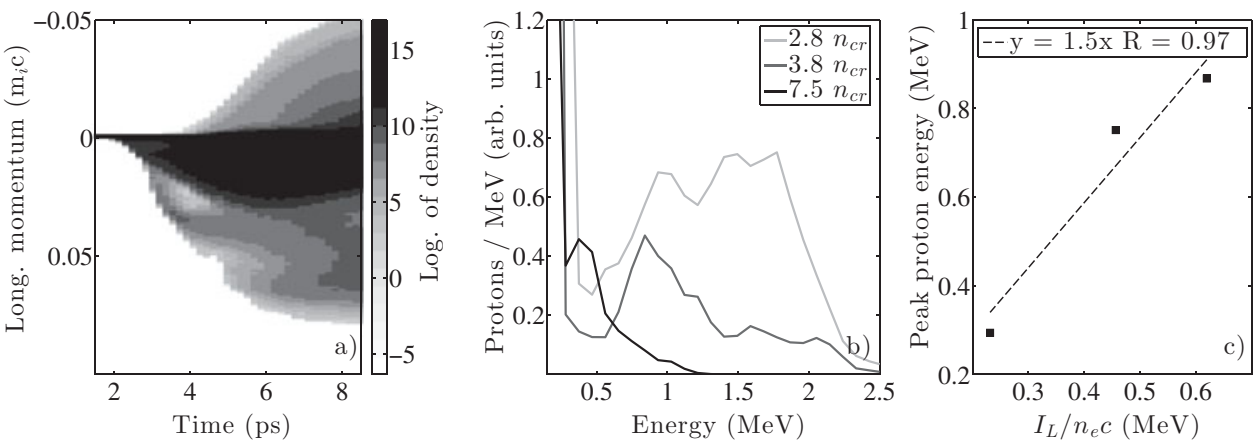

FIGURE 3. (a) Longitudinal proton momentum vs. time for the step-like target with a peak density of $3.8 n_{c r}$. (b) Proton spectra with varying peak target density for an incoming laser of $a_{0}=1.8$ and a step-like density profile. (c) Scaling of the peak proton energy with ratio of laser intensity and peak target density.

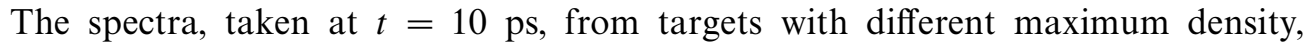
are shown in Fig. 3(b). These indicate increasing peak energy with decreasing target density. The increasing peak energy with decreasing target density is linked to the change in the speed of the front, resulting in a higher velocity of the reflecting potential and therefore higher energy ions.

In the experiment (Palmer et al. 2011), a linear dependency between energy of the proton peaks and $I_{L} / n_{e}$ was observed. Figure 3(c) presents the proton energy for simulations of different target density.

\section{Conclusions}

PIC simulations were performed exploring the effect of the multiple pulse structure of the $\mathrm{CO}_{2}$ laser utilised by Palmer et al. (2011) on the generation of the narrow energy spread proton beams. This pulse structure resulted in a steepening of the target density profile, without which narrow energy spread features would not have been obtained. In contrast to the work of Haberberger et al. (2012), it was found that a step-like density gradient was required to obtain on-axis narrow energy spread ion spectra. The simulations also reveal the peaked proton energy spectra scaled linearly with $I_{L} / n_{e}$, over the parameter range of the study, for targets with a sharpened density profile, supporting the experimentally observed scaling.

\section{Acknowledgements}

The authors would like to acknowledge the assistance of the ATF staff and experimental collaborators in the performance of the experiment and the OSIRIS collaboration for access to the simulation code. Computing services were provided by Imperial College HPC. The work was funded by EPSRC grants EP/E035728/1, EP/K022415/1, STFC grant ST/J002062/1 and DOE grant DE-FG0207ER41488.

\section{REFERENCES}

Bulanov, S. V. and Esirkepov, T. Z. 2002 Oncological hadrontherapy with laser ion accelerators. Phys. Lett. A 299, 240-247. 
Daido, H., Nishiuchi, M. and Pirozhkov, A. S. 2012 Review of laser-driven ion sources and their applications. Rep. Prog. Phys. Phys. Soc. (Great Britain) 75(5), 056401.

Denavit, J. 1992 Absorption of high-intensity subpicosecond lasers on solid density targets. Phys. Rev. Lett. 69(21), 3052-3056.

Dover, N. P. et al. 2012 Optical probing of shocks driven into overdense plasmas by laser hole-boring. arXiv $\mathbf{1 2 0 5 . 4 5 5 8 v}, 7-10$.

Esirkepov, T., Borghesi, M., Bulanov, S., Mourou, G. and Tajima, T. 2004 Highly efficient relativisticion generation in the laser-piston regime. Phys. Rev. Lett. 92(17), 175003.

Fiuza, F., Stockem, A., Boella, E., Fonseca, R. A., Silva, L. O., Haberberger, D., Tochitsky, S., Gong, C., Mori, W. B. and Joshi, C. 2012 Laser-driven shock acceleration of monoenergetic ion beams. Phys. Rev. Lett. 109(21), 215001.

Fonseca, R. A. et al. 2002 OSIRIS: a three-dimensional, fully relativistic particle in cell code for modeling plasma based accelerators. Lecture Notes Comput. Sci. 2331, 342-351.

Haberberger, D., Tochitsky, S., Fiuza, F., Gong, C., Fonseca, R. A., Silva, L. O., Mori, W. B. and Joshi, C. 2012 Collisionless shocks in laser-produced plasma generate monoenergetic high-energy proton beams. Nature Phys. 8(1), 95-99.

Hatchett, S. P. et al. 2000 Electron, photon, and ion beams from the relativistic interaction of Petawatt laser pulses with solid targets. Phys. Plasmas 7(5), 2076.

Hegelich, B. M., Albright, B. J., Cobble, J., Flippo, K., Letzring, S., Paffett, M., Ruhl, H., Schreiber, J., Schulze, R. K. and Fernández, J. C. 2006 Laser acceleration of quasi-monoenergetic MeV ion beams. Nature 439(7075), 441-444.

Key, M. H. 2007 Status of and prospects for the fast ignition inertial fusion concept. Phys. Plasmas 14(5), 055502.

Macchi, A., Borghesi, M. and Passoni, M. 2013 Ion acceleration by superintense laser-plasma interaction. Rev. Mod. Phys. 85(2), 751-793.

Macchi, A., Cattani, F., Liseykina, T. and Cornolti, F. 2005 Laser acceleration of ion bunches at the font surface of overdense plasmas. Phys. Rev. Lett. 94(16), 165003.

Mora, P. 2003 Plasma expansion into a vacuum. Phys. Rev. Lett. 90(18), 185002.

Najmudin, Z. et al. 2011 Observation of impurity free monoenergetic proton beams from the interaction of a CO2 laser with a gaseous target. Phys. Plasmas 18(5), 056705.

Palmer, C. A. J. et al. 2011 Monoenergetic proton beams accelerated by a radiation pressure driven shock. Phys. Rev. Lett. 106(1), 014801.

Palmer, C. A. J. et al. 2012 Rayleigh-Taylor instability of an ultrathin foil accelerated by the radiation pressure of an intense laser. Phys. Rev. Lett. 108(22), 225002.

Pogorelsky, I. V. et al. 2012 Ion acceleration by laser hole-boring into plasmas. AIP Conf. Proc. 1507, 814-819.

Robinson, A. P. L., Gibbon, P., Zepf, M., Kar, S., Evans, R. G. and Bellei, C. 2009 Relativistically correct hole-boring and ion acceleration by circularly polarized laser pulses. Plasma Phys. Control. Fusion 51(2), 024004.

Robinson, A. P. L., Zepf, M., Kar, S., Evans, R. G. and Bellei, C. 2008 Radiation pressure acceleration of thin foils with circularly polarized laser pulses. New J. Phys. 10(1), 013021.

Schreiber, J. et al. 2006 Analytical model for ion acceleration by high-intensity laser pulses. Phys. Rev. Lett. 97(4), 045005.

Schwoerer, H., Pfotenhauer, S., Jäckel, O., Amthor, K.-U., Liesfeld, B., Ziegler, W., Sauerbrey, R., Ledingham, K. W. D. and Esirkepov, T. 2006 Laser-plasma acceleration of quasimonoenergetic protons from microstructured targets. Nature 439(7075), 445-448.

Silva, L. O., Marti, M., Davies, J. R. and Fonseca, R. A. 2004 Proton shock acceleration in laser-plasma interactions. Phys. Rev. Lett. 92(1), 015002.

Snavely, R A. et al. 2000 Intense high-energy proton beams from Petawatt-laser irradiation of solids. Phys. Rev. Lett. 85(14), 2945.

Ter-Avetisyan, S., Schnürer, M., Sokollik, T., Nickles, P. V., Sandner, W., Reiss, H. R., Stein, J., Habs, D., Nakamura, T. and Mima, K. 2008 Proton acceleration in the electrostatic sheaths of hot electrons governed by strongly relativistic laser-absorption processes. Phys. Rev. E 77(1), 016403.

Toncian, T. et al. 2006 Ultrafast laser-driven microlens to focus and energy-select mega-electron volt protons. Science (New York, N.Y.) 312(5772), 410-413. 
Wilks, S. C., Kruer, W. L., Tabak, M. and Langdon, A. B. 1992 Absorption of ultra-intense laser pulses. Phys. Rev. Lett. 69(9), 1383-1386.

Wilks, S. C., Langdon, A. B., Cowan, T. E., Roth, M., Singh, M., Hatchett, S., Key, M. H., Pennington, D., MacKinnon, A. and Snavely, R. A. 2001 Energetic proton generation in ultra-intense lasersolid interactions. Phys. Plasmas 8(2), 542.

Zhang, X., Shen, B., Yu, M. Y., Li, X., Jin, Z., Wang, F. and Wen, M. 2007 Effect of plasma temperature on electrostatic shock generation and ion acceleration by laser. Phy. Plasmas 14(11), 113108.

\section{Culham Prize Winner 2012}

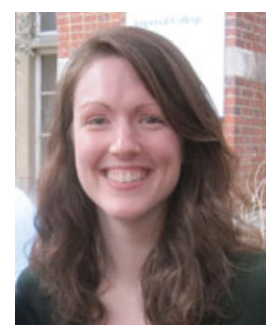

Charlotte Palmer received her undergraduate masters in Physics from the University of Bath in 2007. She then moved to London to take up a PhD position with Prof. Zulfikar Najmudin in the Plasma Group at Imperial College London. The focus of her research was the acceleration of protons and heavier ions through the interaction of high intensity lasers and plasma targets. Charlotte completed her $\mathrm{PhD}$, entitled 'Approaching the radiation pressure regime of proton acceleration with high intensity lasers', in 2012 and was awarded the Plasma Physics Group Culham Thesis Prize 2012. Following her PhD she took up a post-doctoral position at the University of Hamburg in the Helmholtz Young Investigator group of Dr. Jens Osterhoff, where the focus of her research shifted to plasma-based electron acceleration. In 2013 she successfully applied for the Helmholtz Post-doc programme winning a grant to study potential techniques for the diagnosis of ultra-short electron bunches in association with the linear accelerator group at DESY, Hamburg where she is currently employed.

The simulation studies submitted in the paper are a compliment to experimental work, performed during the $\mathrm{PhD}$ at the Accelerator Test Facility of Brookhaven National Laboratory, which was published as an editor's suggestion within Physical Review Letters in 2011. 